\title{
Guest editorial: Acute promyelocytic leukemia: change from "highly fatal to highly curable" leukemia
}

\author{
Norio Komatsu
}

Received: 20 May 2014/ Accepted: 22 May 2014/Published online: 10 June 2014

(c) The Japanese Society of Hematology 2014

Advances have been made in the treatment of acute promyelocytic leukemia (APL) in the past three decades due to the clinical application of all-trans-retinoic acid (ATRA) and arsenic trioxide (ATO) in addition to anthracyclinebased chemotherapy. The use of combination therapy with ATRA and ATO has led to 5-year event-free survival and overall survival rates of approximately $90 \%$. Thus, APL has changed from "highly fatal to highly curable" leukemia, as described by Dr. Li.

Most APL patients have the PML-RAR $\alpha$ fusion gene, which is generated by the reciprocal translocation of the promyelocytic leukemia (PML) gene on chromosome 15 and the retinoic acid receptor $\alpha(\operatorname{RAR} \alpha)$ gene on chromosome 17. The PML-RAR $\alpha$ fusion protein functions as a dominant negative regulator against PML-dependent apoptotic pathways and granulocytic differentiation by the direct transcriptional inhibition of retinoic acid target genes, which blocks cellular differentiation into mature neutrophils. ATRA induces cell growth arrest, granulocytic differentiation, and apoptosis in APL cells via the proteasome-dependent degradation of the PML-RAR $\alpha$ fusion protein and subsequent formation of PML-nuclear bodies (NB). Degradation of the PML-RAR $\alpha$ fusion protein and the reorganization of PML-NB during ATRA treatments are regarded as critical cellular responses, similar to cell growth arrest and apoptosis in leukemia cells. PML also regulates diverse cellular functions including metabolism senescence, apoptosis, autophagy, and angiogenesis. Drs. Nakahara, Weiss, and Ito have focused on the functional

N. Komatsu $(\bowtie)$

Department of Hematology, Juntendo University School of Medicine, 2-1-1 Hongo, Bunkyo-ku, Tokyo 113-8421, Japan e-mail: komatsun@juntendo.ac.jp role of PML in hematopoietic and leukemic stem cell maintenance, and refer to FOXO3A in their study.

FOXO3A is a member of the Forkhead family of transcription factors and is characterized by a highly conserved forkhead domain with a winged-helix motif and measurable DNA-binding activity. This molecule functions as a transcription factor for various vital genes involved in cellcycle regulation (p27/Kip1, p130-Rb2, and cyclin D1/2), apoptosis (Bim, the Fas ligand, and TRAIL), and DNA repair (GADD45a). FOXO3A was recently reported to exert its function as a key molecule for ATRA-induced granulocytic differentiation and apoptosis in APL [1]. In addition, several lines of evidence have shown that not only PML, but also FOXO3A plays important roles in maintaining the hematopoietic stem cell pool [2, 3], and these findings strongly suggest a close relationship between these two molecules in leukemogenesis.

APL is often accompanied by severe disseminated intravascular coagulation (DIC), which results in lifethreatening hemorrhaging. The mortality rate of APL patients was previously very high due to massive bleeding from vital organs such as the brain, lungs, and gastrointestinal tract. Although the introduction of ATRA into induction chemotherapy has markedly improved the overall survival rates of APL patients, more than $10 \%$ of patients still die from severe hemorrhaging. Therefore, the management of hemorrhaging has yet to be successfully achieved. Dr. Ikezoe reviewed the pathogenesis and management of DIC caused by APL. In addition, he referred to the therapeutic efficacy of recombinant human soluble thrombomodulin for the management of DIC in APL patients.

Thus, ATRA markedly improved the complete remission rate and significantly prolonged overall survival in APL patients. ATO is also used as a first-line treatment in 
APL patients in combination with ATRA. However, several issues still remain to be resolved including overcoming resistance to ATRA and ATO, efficiently controlling the coagulopathy of APL patients, and the application of differentiation therapy to other types of acute myelogenous leukemias. We recently reported that the inhibition of SIRT2 deacetylase activity induced granulocytic differentiation in the APL cell line, NB4 [4]. This finding was also observed in HL-60, which does not possess PML-RAR $\alpha$. Although the concept is still speculative, our result suggests that targeting SIRT2 is a viable strategy to induce leukemic cell differentiation. A clearer understanding of the mechanisms underlying APL differentiation may provide an insight into the eradication of non-APL leukemic cells.

\section{References}

1. Sakoe Y, Sakoe K, Kirito K, Ozawa K, Komatsu N. FOXO3A as a key molecule for all-trans retinoic acid-induced granulocytic differentiation and apoptosis in acute promyelocytic leukemia. Blood. 2010;115:3787-95.

2. Ito K, Bernardi R, Morotti A, Matsuoka S, Saglio G, Ikeda Y, et al. PML targeting eradicates quiescent leukaemia-initiating cells. Nature. 2008;453:1072-8.

3. Miyamoto K, Arai KY, Naka K, Arai F, Takubo K, Yamazaki S, et al. Foxo3a is essential for maintenance of the hematopoietic stem cell pool. Cell Stem Cell. 2007;1:101-12.

4. Sunami Y, Araki M, Hironaka Y, Morishita S, Kobayashi M, Liew $\mathrm{EL}$, et al. Inhibition of the NAD-dependent protein deacetylase SIRT2 induces granulocytic differentiation in human leukemia cells. PLoS One. 2013;8:e57633. 